\title{
"ANÁLISE DINÂMICA DE VIGAS DE EIXO CURSO E SEÇÃO ABERTA SUBMETIDAS A CARGAS MÓVEIS ${ }^{a "}$
}

\author{
GILBERTO CARBONARI
}

\section{RESUMO}

Fundamentalmente, o presente trabalho faz uma análise linear de pontes ou vigas curvas assimétricas de seção transversal aberta e de parede fina, com propriedades físicas, geométricas e raio de curvatura constantes ao longo do eixo baricêntrico. Para tanto, utilizaram-se as equações diferenciais de VLASOV considerando o acoplamento entre as deformações nas direções vertical, transversal, axial e torcional. Na solução do sistema de quatro equações com derivadas parciais foi utilizado um apropriado método numérico de integração (Diferenças Finitas Centrais). A análise divide-se, basicamente, em dois tipos: análise DINÂMICA e ESTÁTICA. Ambas são utilizadas também na determinação do coeficiente de impacto (C.M.D.J. A primeira refere-se tanto à determinação das características dinâmicas básicas (freqüências naturais e respectivos modos de vibração), como também à determinação da resposta dinâmica da viga, em tensões e deformações, para cargas móveis arbitrárias. Vigas com qualquer combinação das condições de contorno, incluindo bordos rotulados e engastados nas direções de flexão e na torção, são consideradas. Os resultados da análise teórica, obtidos pela aplicação de programas computacionais implementados em microcomputador (análise estática) e no computador B-67Q0 (análise dinâmica), são comparados tanto com os da bibliografia técnica como também com resultados experimentais, apresentando boa correlação.

PALAVRAS-CHAVE: Vigas Curvas, Cargas Móveis, Freqüências Naturais. 


\section{1 - INTRODUÇÃO}

Novas soluções às crescentes necessidades do transporte rodoviário e ferroviário, especialmente em ambientes urbanos, implicam freqüentemente no uso de vias elevadas. A periodicidade dos apoios resulta na possibilidade de comportamento dinâmico insatisfatório, efeito que adquire particular importância em sistemas de alta velocidade. Modelos teóricos que permitam o estudo do acomplamento entre veículo e via devem então ser desenvolvidos. Visando esse objetivo é estudado no presente trabalho o comportamento estático e dinâmico de vigas curvas de parede fina e seção aberta, incluindo-se a resposta das mesmas a sistemas de cargas em movimento. Devido à necessidade de implementação de soluções que permitissem inicialmente a realização de estudos paramétricos a baixo custo, e posteriormente a sua incorporação num modelo de interação, a estrutura da via foi idealizada como viga. A escolha de um modelo unidimensional da via implica numa simplificação importante, pela qual não podem ser levadas em conta, por exemplo, possíveis distorções da seção transversal. Em contrapartida, uma representação mais apurada da via poderia conduzir a custos computacionais inadmissíveis e, possivelmente, desnecessários.

São em conseqüência adotadas como ponto de partida as equações de movimento propostas por $\operatorname{VLASOV}^{10}$ para a viga curva em planta com seção arbitrária aberta de parede fina. As quatro equações diferenciais ordinárias de Vlasov, aplicáveis a vigas elásticas com raio de curvatura constante, são resolvidas através de uma formulação de diferenças finitas centrais. Foram obtidas: a) a resposta estática; b) as características dinâmicas básicas (frequiências e modos naturais de vibração), e c) resposta dinâmica à ação de cargas móveis.

As diversas soluções são comparadas com resultados disponíveis na literatura técnica, incluindo valores experimentais, verificando-se assim a precisão e confiabilidade do método. Com base nos exemplos estudados são finalmente estabelecidas curvas para a determinação prática da freqüência fundamental de pontes de concreto de planta curva.

\section{2 -EQUAÇÕES DE MOVIMENTO E MÉTODOS DE SOLUÇÃO}

Considere-se a viga curva circular de paredes finas e seção aberta, com propriedades constantes ao longo da coordenada $\mathrm{s}$ que define o comprimento de arco medido a partir do extremo de viga, como se ilustra na Fig. 2.1. Seja $\mathrm{R}$ o raio de curvatura no plano normal ao eixo vertical $\mathrm{Y}$. A teoria proposta por Valsov está baseada nas seguintes hipóteses:

- O material é elástico e linear

- A espessura das paredes é pequena em relação às outras dimensões da seção tranversal, sendo as dimensões da última pequenas em relação ao comprimento

- A seção transversal não é deformável, isto é, é rígida no seu plano, mas pode empenar livremente
- As deformações devidas às tensões de cisalhamento na superfície média da viga são desprezíveis.

Considerando também que a viga apresenta amortecimento de tipo viscoso ou de Newton, resultam as seguintes quatro equações acopladas de movimento:

$$
\begin{aligned}
& E\left(I_{z}+\frac{I_{\omega}}{R^{2}}\right) \frac{\partial^{4} y}{\partial s^{4}}-\frac{G K_{t}}{R^{2}} \frac{\partial^{2} y}{\partial s^{2}}+\frac{E I_{w}}{R} \frac{a^{4} \theta}{\partial s^{4}}-\frac{\left(E I_{z}+G K_{t}\right)}{R} \frac{\partial^{2} E}{\partial s^{2}}+ \\
& +\rho A\left(\frac{\partial^{2} y}{\partial z^{2}}-a_{z} \frac{\partial^{2} \theta}{\partial t^{2}}\right)-\rho Y_{z}\left(\frac{\partial^{4} y}{\partial s^{2} \partial t^{2}}-\frac{1}{R} \frac{\partial^{2} \theta}{\partial t^{2}}\right)+\beta A \alpha \frac{\partial Y}{\partial t}= \\
& =r_{1}(s, t) \\
& \mathbb{E} I_{Y}\left(\frac{\partial^{4} z}{\partial s^{4}}+\frac{1}{B} \frac{\partial^{2} z}{\partial s^{2}}\right)+\frac{E A}{R}\left(\frac{Z}{R}+\frac{\partial x}{\partial s}\right)+\partial A\left(\frac{\partial^{2} z}{\partial t^{2}}+\Delta \frac{\partial^{2} \dot{\partial}}{\partial t}\right)- \\
& \text { - } \rho \operatorname{Ar}_{Y}^{2}\left(\frac{\partial^{4} z}{\partial s^{2} \partial t^{2}}+\frac{1}{R^{2}} \frac{\partial^{2} z}{\partial t^{2}}\right)+\rho A \alpha \frac{\partial z}{\partial t}=P_{z_{i}} \text { (s, t) (2.2) } \\
& E I_{d k} \frac{\partial^{4} \theta}{\partial s^{4}}+\frac{E I_{\partial^{2}}}{R} \frac{\partial^{4} y}{\partial s^{4}}-\frac{\left(E I_{z}+G K_{t}\right)}{R} \frac{\partial^{2} y}{\partial s^{2}}-G K_{t} \frac{\partial^{2} \theta}{\partial s^{2}}+\frac{E I z}{R^{2}} g+ \\
& +\Gamma A\left(r^{2} \frac{\partial^{2} \theta}{\partial t^{2}}+a_{y} \frac{\partial^{2} z}{\partial t^{2}}-a z \frac{\partial^{2} \gamma}{\partial t^{2}}\right)-p I_{\omega}\left(\frac{\partial^{4} \theta}{\partial z^{2} a t^{2}}+\frac{1}{R} \frac{\partial^{4} y}{\partial s^{2} \partial t^{2}}\right)+ \\
& +\rho B \Sigma^{2} \otimes \frac{\partial \theta}{\partial t}=M_{t_{i}}(s, t) \\
& -\frac{E I y}{R}\left(\frac{\partial^{3} z}{\partial s^{3}}+\frac{1}{R^{2}} \frac{\partial z}{\partial s}\right)-E A\left(\frac{\partial^{2} x}{\partial s^{2}}-\frac{1}{R} \frac{\partial z}{\partial s}\right)+D A \frac{\partial^{2} x}{\partial t^{2}}-\rho A \alpha \frac{\partial x}{\partial t}= \\
& =P_{x_{i}}(s, t)
\end{aligned}
$$

onde,

$x(s, t), y(s, t), z(s, t)$ - deslocamentos nas direçôes axial, vertical e lateral, respectivamente;

$\theta(\mathrm{s}, \mathrm{t})$ - gíro da seção transversal ao redor do centro de flexão $A_{0}$;

$P_{x_{i}}(s, t), P_{y_{j}}(s, t), P_{z_{i}}(s, t)$ - projeçóes das cargas externas nas direçōes $x, y$ e 2 , respectivamente, por unidade de comprimento;

$\mathbf{M}_{\mathbf{t}_{\mathrm{i}}}(\mathrm{s}, \mathrm{t})$ - momento torçor devido às cargas extertras, em tomo do centro de flexão Ao, por unidade de comprimento;

E - módulo de Young do material; $1 \quad \mathbf{E}$

$\mathrm{G}=\quad-$ módulo de elasticidade transversal $2(1+v)$ do material;

$\alpha-$ coeficiente de amortecimento;

$v$ - coeficiente de Poisson do material da viga;

A - área da seçăo transversal; 
$\rho$ - massa específica do material da viga;

$R$ - raio de curvatura;

$\mathrm{GK}_{\mathrm{t}}$ - rigidez torcional da seçåo;

$\mathrm{K}_{\mathrm{t}}$ - constante de torção de St. Venant;

$\mathbf{I}_{\mathrm{y}}, \mathbf{I}_{\mathbf{z}}$ - momento de inércia com relaçāo aos eixos principais y e $z$, respectivamente.

$I_{\omega}=\int_{A} \phi^{2} \mathrm{dA}$ - constante de empenamento (momento setorial de ínércia);

$\phi$ - coordenada setorial normalizada;

$a_{y}, a_{z}$ - coordenadas do centro de flexão $\mathbf{A}_{0}$ tomadas ao longo dos eixos princípais y e z (Fig. 2.1.), respectivamente;

$I_{y}^{2}=I_{y} / A, \quad I_{z}^{2}=I_{z} / A-$ raio de giraçăo com relaçāo aos eixos principais y e $\mathrm{z}$;

$\mathrm{r}_{\omega}^{4}=\mathrm{I}_{\omega} / \mathrm{A}$

$r^{2}=a_{y}^{2}+a_{z}^{2}+r_{y}^{2}+r_{z}^{2}$.

$\mathrm{Na}$ implementação da solução de eqs. (2.1) a (2.4) verificou-se que o sistema não é auto-adjunto e consequentemente conduz a coeficientes de rigidez e de massa não simétricos. Foram introduzidas então algumas modificações, admitindo-se que os termos

$\left.\frac{\rho I_{z}}{R} \frac{\partial^{2} j}{\partial t^{2}}, \frac{\rho I_{\omega}}{R}-\frac{\partial^{4} y}{\partial s^{2}} \frac{E t^{2}}{\partial t^{2}}, \frac{\partial^{3} z}{\partial s^{3}}+\frac{1}{R^{2}} \frac{\partial z}{\partial s}\right)$

são nulos; sem afetar a precisão dos resultados.

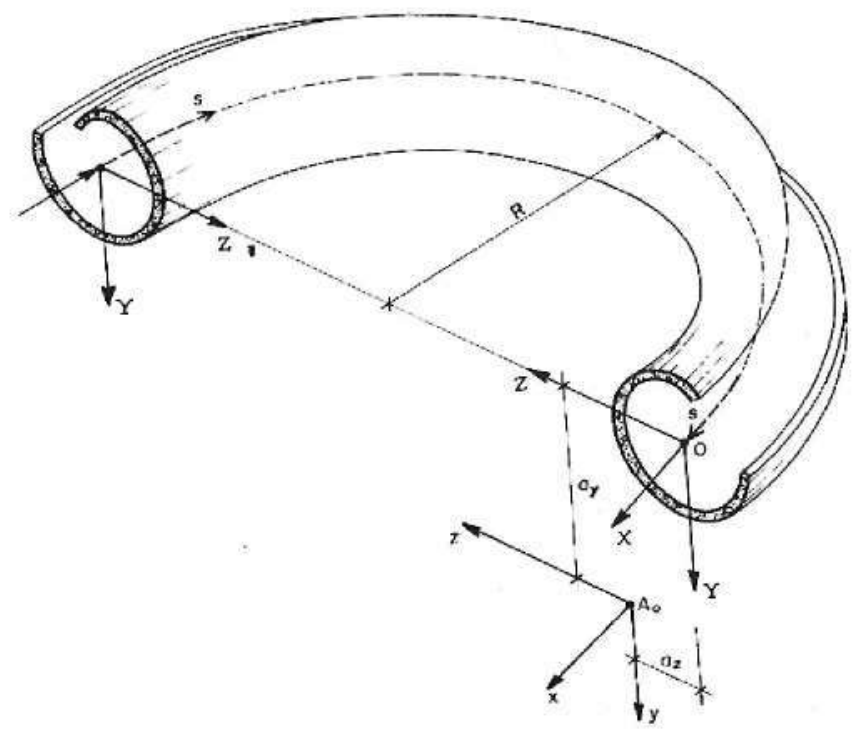

Fig. 2.1 - Características geométricas e notação da viga curva de seção aberta e parede fina

Entre os vários métodos de solução de equações diferenciais existentes, optou-se pela aplicação de diferenças fínitas centrais (DFC). Os operadores diferenciais nas eqs, (2.1) a 2.4) e nas condições de bordo são substituídos por operadores em diferenças finitas, isto é, podem ser expressos em termos das ordenadas das funçốs de desloca. mento em pontos discretos de coordenadas $\mathrm{j} \measuredangle(\mathrm{c}=1, \ldots \mathrm{N})$. Para cargas estáticas, as derivadas com respeito ao tempo são nulas, sendo as açбes extemas independentes de $t$. Obtem-se nesse caso um sistema de equaçōes algébricas nos $4(N-2)$ deslocamentos desconhecidos, da forma:

$$
\text { IK } \overrightarrow{\mathbf{U}}=\overrightarrow{\mathbf{P}}
$$

onde $\mathbf{K}$ equivale à matriz de rigidez global da viga, $\vec{U}$ é o vetor de deslocamentos a giros e $\overrightarrow{\mathbf{P}}$ o vetor de cargas externas. Já a determinação das propriedades dinâmicas é feita a partir das equaçđes homogêneas, isto 6 , toma-se $\mathbf{P}_{\mathbf{y}}, \mathbf{P}_{\mathbf{z}}$, $M_{t}$ e $P_{X}$ igual a zero nas eqs. (2.1) a (2.4), nas quais o amortecimento também é considerado nulo. Soluçōes da forma:

$$
\begin{aligned}
& y(s, t)=y n(s) \operatorname{sen} \omega_{n} t \\
& z(s, t)=z_{n}(s) \operatorname{sen} \omega_{n} t \\
& \theta(s, t)=\theta_{n}(s) \operatorname{sen} \omega_{n} t \\
& x(s, t)=x_{n}(s) \operatorname{sen} \omega_{n} t
\end{aligned}
$$

conduzem, após a posterior aplicação de DFC, a um problema linear de autovalores:

$$
\left(\mathrm{IK}-\omega_{\mathrm{n}}^{2} \mathrm{MM}\right) \overrightarrow{\mathbf{U}}_{\mathrm{HI}}=0
$$

o qual é resolvido pelo método de interação por subespaços, implementado no programa DINAUT.

Finalmente, a solução das equações de movimento para cargas móveis é obtida através de um esquema misto de integração numérica direta simultânea nas variáveis espacial s e temporal t. 0 procedimento é implícito, exigindo a solução de um sistema de equações algébricas a cada passo de integração t. O método de integração, embora só condicionalmente estável, é muito eficiente no problema em estudo, pois a descrição apurada das cargas móveis usualmente requer a adoção de intervalos de integração da mesma ordem que $\mathrm{t}_{\mathrm{c} r} \mathrm{O}$ esquema, que inclui como nos casos anteriores a geração automática das matrizes do sistema para apoios rotulados ou fixos, foi implementado no programa VIGADIN.

Em qualquer caso, a partir dos deslocamentos e giros calculados, torna-se possível a obtenção das forças internas nas viga, dadas por:

$$
\begin{aligned}
& \text { na viga, dadas por: } \\
& \mathbf{N}_{x}=\text { EA }\left(\frac{\partial x}{\partial s}-\frac{z}{R}\right), \\
& M_{y}=E I_{y}\left(\frac{\partial^{2} z}{\partial s^{2}}+\frac{z}{R}\right), \\
& M_{z}=-E I_{z}\left(\frac{\partial^{2} y}{\partial s^{2}}-\frac{\theta}{R}\right),
\end{aligned}
$$




$$
\begin{aligned}
& T_{s}=G K_{t}\left(\frac{\partial \theta}{\partial s}+\frac{1}{R} \frac{\partial y}{\partial s}\right), \\
& T_{\theta}=-E I_{\omega}\left(\frac{\partial^{3} \theta}{\partial s^{3}}+\frac{1}{R} \frac{\partial^{3} y}{\partial s^{3}}\right), \\
& T=T_{s}+T_{\theta}, \\
& B_{M}=-E I_{\omega}\left(\frac{\partial^{2} \theta}{\partial s^{2}}+\frac{1}{R} \frac{\partial^{2} y}{\partial s}\right), \\
& Q_{y}=-E I_{z}\left(\frac{\partial^{3} y}{\partial s^{3}}-\frac{1}{R} \frac{\partial \theta}{\partial s}\right)+\frac{T}{R}, \\
& Q_{z}=-E I_{y}\left(\frac{\partial^{3} z}{\partial s^{3}}+\frac{1}{R^{2}} \frac{\partial z}{\partial s}\right) x .
\end{aligned}
$$

onde $\mathrm{N}_{\mathrm{x}}$ denota a força axial; $\mathbf{M}_{\mathrm{y}}$ e $\mathbf{M}_{\mathrm{z}}$ os momentos fletores internos com respeito aos eixos $\mathrm{Y}$ e $\mathrm{Z} ; \mathrm{T}_{\mathrm{S}}$ o momento torçor de St. Venant; $T_{\theta}$ o momento de empenamento; $\mathrm{T}$ o momento torçor total; $\mathrm{B}_{\mathrm{M}}$ o bimomento; $\mathrm{Q}_{Y}$ e $\mathrm{Q}_{Z}$ os cortantes internos com repeito aos eixos $\mathrm{Y}$ e $\mathrm{Z}$, respectivamente. As forças internas podem ser calculadas em pontos discretos $s j=j \Delta s$, após substituir nas eqs. (2.8.a) a (2.8.i) os operadores diferenciais pelas correspondentes expressöes em DFC.

Detalhes sobre os operadores diferenciais e a forma das matrizes Ik e IM assim como a distribuição de cargas móveis podem ser observados na Ref. [2].

\section{3 - PROPRIEDADES DINÂMICAS DE VIGAS DE SE- ÇÃO ABERTA}

A comparação de modos e freqüências naturais de vigas curvas de seção aberta, calculados mediante o emprego de DINAUT, com valores obtidos por outros autores usando procedimentos teóricos ou experimentais é de fundamental importância e será examinada a seguir. O número de trabalhos sobre o tema encontrados na literatura técnica é muito reduzido, e limitado a vigas de um só vão.

CULVER $^{4}$ calculou em 1967 as freqüências naturais de vigas curvas horizontais com seção prismática e dupla simetria, considerando apenas vibrações normais ao plano de curvatura. SHORE \& CHAUDHURI ${ }^{9}$ estudaram posteriormente as vibrações livres de vigas curvas horizontais incorporando a rotação à formulação do problema. Já na presente década, YOO \& FEHRENBACH ${ }^{11}$ incluíram também a contribuição do empenamento, efeitos da inércia rotatória com respeito à flexão e à torção, e os efeitos da anti-simetria da seção trasnversal, através de uma formulação em Elementos Finitos. As hipóteses básicas de Yoo e Fehrenbach são essencialmente idênticas às aceitas no presente trabalho.

Exemplo A: O primeiro exemplo a ser considerado é um conjunto de vigas curvas birotuladas de $512,27 \mathrm{~cm}$ de comprimento. O ângulo interno varia de 10 até 90 graus.
Propriedades relevantes são: $\mathrm{E}=200,1 \mathrm{GN} / \mathrm{m}^{2}, \mathrm{G}=77,3$ $\mathrm{GN} / \mathrm{m}^{2}, \rho=7800 \mathrm{~kg} / \mathrm{m}^{3}, \Lambda=92,9 \mathrm{~cm}^{2}, \mathrm{I}_{\mathrm{z}}=11362 \mathrm{~cm}^{4}$, $\mathrm{I}_{\mathrm{y}}=3871 \mathrm{~cm}^{4}, \mathrm{I}_{\omega}=555878 \mathrm{~cm}^{6}, \mathrm{~K}_{\mathrm{t}}=1470,85 \mathrm{~cm}^{4} \cdot \mathrm{Na}$ Tabela 3.1, as frequências naturais determinadas mediante o programa DINAUT são comparadas com as calculadas

\begin{tabular}{|c|c|c|c|c|c|}
\hline $\begin{array}{l}\text { Raio } \\
\text { (m) }\end{array}$ & $\begin{array}{l}\text { Angulo } \\
\text { Interno } \\
\text { (graus) }\end{array}$ & $\begin{array}{l}\text { DINAUT } \\
19 \text { nós }\end{array}$ & CULVER & $\begin{array}{c}\text { SHORE \& } \\
\text { CHAUDHURI }\end{array}$ & $\begin{array}{c}\text { YOO \& } \\
\text { FEHRENBACH }\end{array}$ \\
\hline 29,35 & 10 & 202,7 & 202,5 & 203,3 & 204,7 \\
\hline 14,68 & 20 & 184,4 & 184,3 & 186,3 & 190,2 \\
\hline 9,78 & 30 & 162,2 & 162,2 & 164,7 & 165,8 \\
\hline 7,34 & 40 & 140,5 & 140,0 & 142,8 & 141,7 \\
\hline 5,87 & 50 & 120,9 & 120,9 & 122.8 & 121,3 \\
\hline 4,89 & 60 & 103,8 & 103,8 & 105,2 & 103,9 \\
\hline 4,19 & 70 & 88,9 & 88,9 & 90,0 & 89,6 \\
\hline 3,67 & 80 & 75,9 & 76,0 & 76.2 & 76,8 \\
\hline 3,26 & 90 & 64,6 & 64,6 & 65,2 & 64,0 \\
\hline
\end{tabular}
por CUI.VER ${ }^{4}$, SHORE \& CHAUDHURI ${ }^{9}$, YOO \&FEH$\mathrm{RENBACH}^{11}$. $\hat{\mathrm{E}}$ observada uma excelente correlação, sendo que as diferenças percentuais ficam ao redor de $1 \%$.

Tabela 3.1 - Frequências naturais (radiseg) da viga birotulada Curva em flexo-torção. (Exemplo A)

Exemplo B: O próximo exemplo corresponde a uma viga reta (curvatura nula) elevada pertencente a um sistema de transporte urbano com 25 metros de comprimento, cujas condições de contorno sâo: birotulada na flexão vertical, birotulada na flexăo horizontal e biengastada na torçāo. Outras propriedades são as seguintes: $\mathrm{E}=35,5$ $\mathrm{GN} / \mathrm{m}^{2} ; \mathrm{G}=14,792 \mathrm{GN} / \mathrm{m}^{2} ; \rho=2500 \mathrm{~kg} / \mathrm{m}^{3} ; \mathrm{A}=1,085$ $\mathrm{m}^{2} ; \mathrm{I}_{\mathrm{Y}}=0,4722 \mathrm{~m}^{4} ; \mathrm{I}_{\mathrm{Z}}=0,312 \mathrm{~m}^{4} ; \mathrm{I}_{\omega}=0,1975 \mathrm{~m}^{6}$; $\mathrm{K}_{\mathrm{t}}=0,0105 \mathrm{~m}^{4} ; \mathrm{a}_{\mathrm{y}}=1,273 \mathrm{~m} ; \mathrm{a}_{\mathrm{z}}=0 ; v=0$. A frequên-

\begin{tabular}{|c|c|c|c|c|}
\hline & M o d o & $\begin{array}{l}\text { Flemen- } \\
\text { tos Fini- } \\
\text { tos }[5]^{*} \\
270 \text { nós }\end{array}$ & $\begin{array}{l}\text { DINAUT } \\
19 \text { nós }\end{array}$ & Dif. ( \%) \\
\hline 1 & $\begin{array}{l}\text { - Flexo-Torção } \\
\text { Lateral }\end{array}$ & 4,85 & 4,84 & 0,68 \\
\hline 2 & - Flexão Vertical & $4,92^{* *}$ & 5,05 & 2,65 \\
\hline 3 & $\begin{array}{l}\text { - Flexo-Torção } \\
\text { Lateral }\end{array}$ & 11,91 & 14,77 & 19,40 \\
\hline 4 & $\begin{array}{l}\text {-. Flexo-Torção } \\
\text { Lateral }\end{array}$ & 14,35 & 15,22 & 5,68 \\
\hline 5 & - Flexāo Vertical & 18,40 & 19,93 & 7,68 \\
\hline
\end{tabular}
cia natural da viga em flexão vertical no segundo modo foi determinada experimentalmente. Os resultados estăo indicados na Tabela 3.2.

Tabela 3.2 - Frequências naturais $(\mathrm{Hz})$ da viga reta elevada de um sistema de transporte urbano. (Exemplo B)

* Utilizou-se 234 elementos retangulares de tipo casca polié* drica.

*** Resultado experimental.

Para o primeiro e segundo modos, verificou-se uma ótima correlação com a solução da referência [ 7 ] e com o valor experimental, inclusive com a freqüência medida. A diferença mais acentuada nos modos superiores é atribuída à inadequada representação das condições de contorno na tor- 
ção, visto que, na realidade, os apoios constituem apenas engastamento parcial da seção transversal.

Exemplo C: No exemplo que segue será estudado o caso de uma viga curva horizontal e vão simples de comprimento $40,64 \mathrm{~m}$. As propriedades da seção transversal da viga são: $A=94 ; 8 \mathrm{~cm}^{2}, I_{z}=16440 \mathrm{~cm}^{4}, I_{y}=2347,4 \mathrm{~cm}^{4}$, $\mathrm{Iw}=504855,2 \mathrm{~cm}^{6}, \mathrm{~K}_{\mathrm{t}}=74,5 \mathrm{~cm}^{4}, \mathrm{a}_{\mathrm{y}}=0 \mathrm{e} \mathrm{a}_{\mathrm{z}}=0$. Os módulos de elasticidade longitudinal e transversal são $\mathrm{E}=200,1 \mathrm{GN} / \mathrm{m}^{2}$ e $77,3 \mathrm{GN} / \mathrm{m}^{2}$. A viga possui dois bordos engastados. O raio de curvatura é $38,811 \mathrm{~m}$, e o ângulo interno de 60 graus. Os resultados obtidos da análise são mostrados na Tabela 3.3.

Tabela 3.3 - Freqüência natural ( $\mathrm{rad} / \mathrm{seg})$, viga curva biengastada. (Exemplo C)

$\begin{array}{ccc}\text { DINAUT } & \text { YOO \& } & \text { Dif. (\%) } \\ \text { (1 } 20 \text { nós) } & \text { FEHERENBACH } & \\ 4,150 & 4,1879 & 0,90\end{array}$

Exemplo D: O exemplo que segue, analisado por CHRISTIANO $^{3}$ corresponde a um modelo experimental de uma ponte curva com vão simples, cuja seção transversa! está esquematízada na Fig. 3.1.

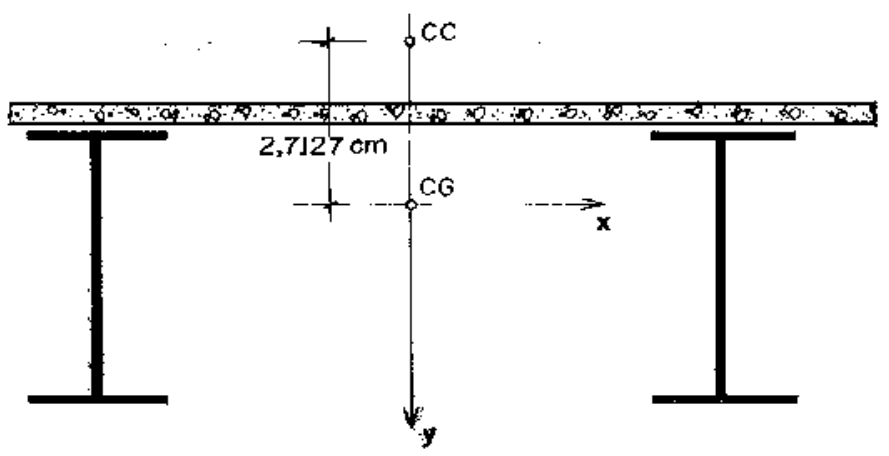

Fig. 3.1 - Seçảo Transversal do modelo da ponte.

- (Exemplos De E)

O modelo possui comprimento de $2,3876 \mathrm{~m}$ e um rato de curvatura com $2,4384 \mathrm{~m}$, sendo o angulo interno de 56 graus. Outras propriedades da viga são: $A=19,758 \mathrm{~cm}^{2}$, $I_{z}=77,3358 \mathrm{~cm}^{4}, I_{y}=913,628 \mathrm{~cm}^{4}, K_{t}=1,9646 \mathrm{~cm}^{4}$, $\mathbf{I}_{\omega}=4524,83 \mathrm{~cm}^{6}, \mathrm{E}=277219 \mathrm{~N} / \mathrm{cm}^{2}, \mathrm{G}=103026 \mathrm{~N} /$ $\mathrm{cm}^{2}, \rho=0,001218 \mathrm{~kg} / \mathrm{cm}^{3}, \mathrm{a}_{y}=-2,7127 \mathrm{~cm}$. Segundo Christiano, as condiçôes de contorno são rotuladas em ambos extremos. A frequência fundamental medida no mo. delo foi de $4,7 \mathrm{~Hz}$, enquanto a computada por Christiano foi de $2,95 \mathrm{~Hz}$. Portanto, verificou-se uma diferença significativa entre a frequência fundamental medida e a calculada. Utilizando o programa DINAUT, a frequència fundamental para bordos birotulados tanto na flexão como na torção foi de $2,928 \mathrm{~Hz}$, com uma diferença de $0,77 \%$ em relação à calculada na Ref. [ 3 ]. Examinando mais detidamente o modelo, porém, chega-se à conclusão que as condiçôes de contorno na torção poderiam ser biengastadas. A frequência fundamental calculada pelo programa DINAUT resulta então de $4,43 \mathrm{~Hz}$, muíto próxima da me- dida no modelo $(4,7 \mathrm{~Hz})$. Nesse exemplo, fica clara a importância das condiçôes de contorno assumidas nos estudos teóricos.

\section{4 - SOBRE A FREQUENCIA FUNDAMENTAL DE PONTES DE CONCRETO}

Um trabalho de muito interesse para o Engenheiro projetista foi realizado por CANTIENE ${ }^{1}$, que determinou as frequencias fundamentais de 224 pontes escolhidas com base nos seguintes critérios: a) eliminação dos valores de estruturas em balanço; b) limitação do raio de curvatura da ponte: $R \geqslant 900 \mathrm{~m}$ (aproximadamente reta) c) limitação do ângulo de inclinaçāo: $\gamma \leqslant 15$ graus); d) limitação da constante de amortecimento $70 \mathrm{KN} / \mathrm{mm} \leqslant \mathrm{K} \geqslant 270$ $\mathrm{KN} / \mathrm{mm}$; e) eliminaçăo dos resultados que não foram obtidos das mediçðes do vão máximo.

A correlação entre a frequência fundamental e o vão pode ser apreciada na Fig. 4.1, a qual mostra também urna relação empírica obtida por regressão.

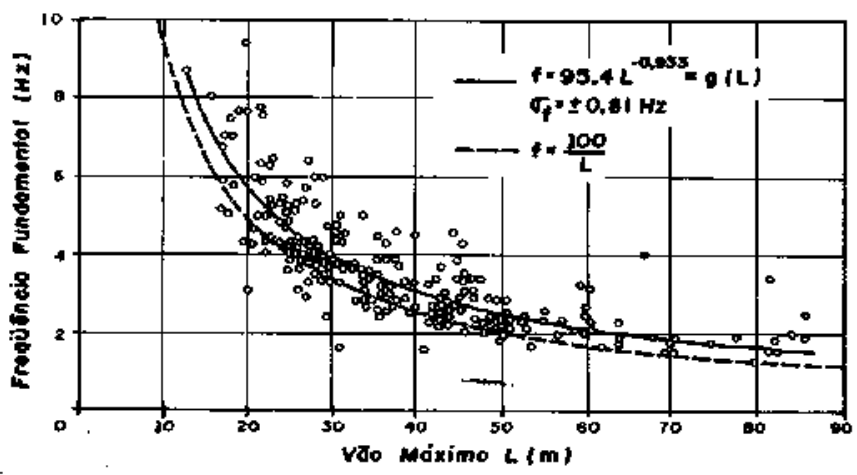

Fig. 4.I - Frequência fundamental f como funçāo do vāo máximo $L$ para 224 valores medidos; $\sigma \mathbf{f}=$ desvio padrão (Ret. [ I D

A partir de resultados de obtenção numérica com DINAUT e dos valores medidos em pontes publicados por Cantiene foram desenvolvidas as curvas da frequencia fundamental de pontes em funçāo do vāo máximo, para ângulos internos que variam de 0 atê 80 graus, representadas na Fig. 4.2.

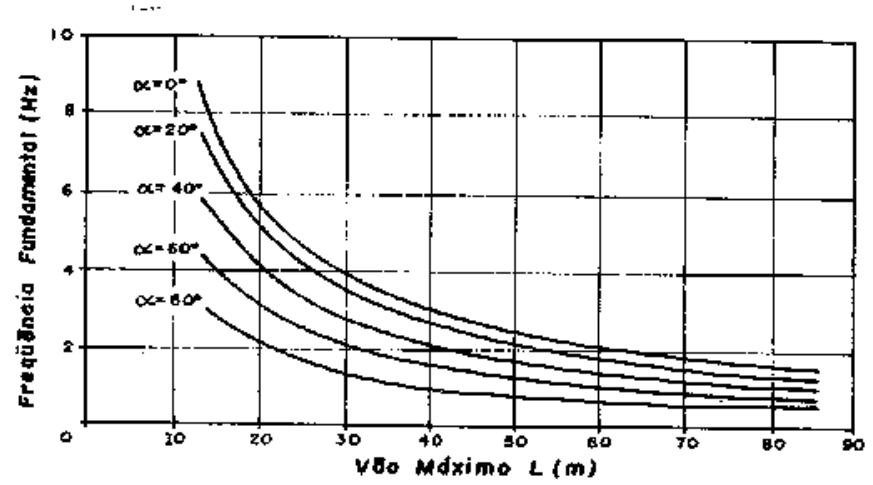

Fig. 4.2 - Frequência fundamental de pontes retas e curvas em funç̧̃ăo do vão máximo $L$. 


\section{5 - ANÁlise DINÂMICA DE VIGAS CURVAS SOB VARGAS MÓVEIS}

Com o duplo objetivo de verificar a validade das equações diferenciais utilizadas na análise dinâmica da viga curva e a precisão do método numérico empregado na solução dessas equações, serão comparados valores obtidos por meio de VIGADIN com resultados teóricos e experimentais existentes na literatura.

Exemplo E: O primeiro exemplo a ser analisado é apresentado por CHRISTIANO ${ }^{3}$. As propriedades dinâmicas da viga curva, mostrada na Fig. 5.1, foram estudadas na Seção 3 (Exemplo D), que inclui também as dimensões e propriedades da mesma. Ambos apoios são rotulados, tanto na flexão horizontal e vertical, como também na torção. CHRISTIANO ${ }^{3}$ determinou experimentalmente o efeito de uma carga vertical de $60 \mathrm{~N}$, que se desloca sobre a viga com velocidade constante de $4 \mathrm{~m} / \mathrm{s}$.

CHRISTIANO $^{3}$ expressa a resposta dinâmica do modelo em termos do Coeficiente de Majoração Dinâmica (C.M.D.), definido como a razão entre a resposta dinâmica e o deslocamento vertical estático no centro do vão. Portanto, a comparação entre os resultados obtidos por CHRIS$\mathrm{TIANO}^{3}$ e os do presente trabalho, só é possível depois de conhecida a resposta estática da viga.

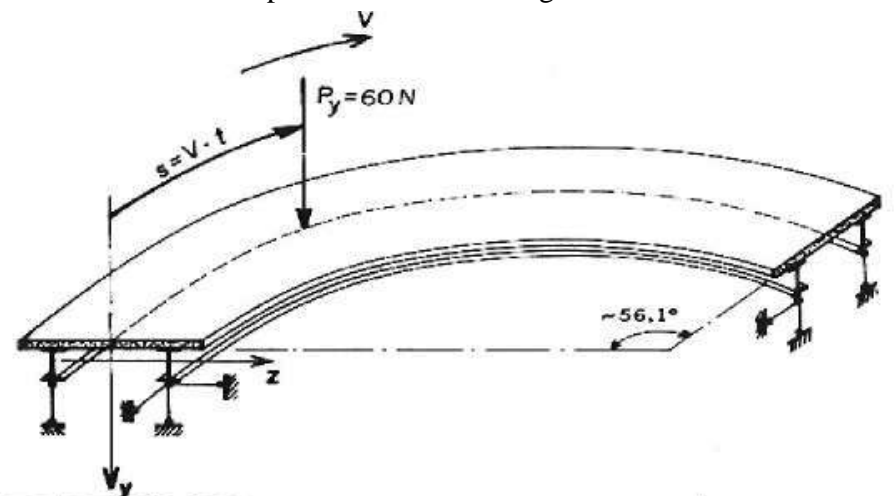

Fig. 5.1 - Viga (Ponte) curva horizontal sujeita à carga em movimento com velocidade constante V. (Exemplos D e E)

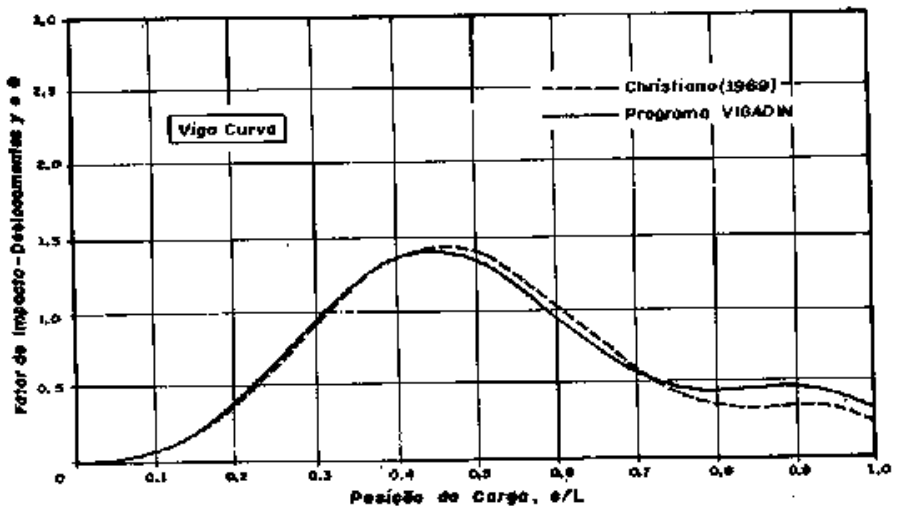

Fig. 5.2 - Valores do fator de impacto (C.M.D.)

Exemplo F: Será analisado, neste exemplo, o comportamento dinâmica da viga reta elevada do exemplo B.
Os trens de cargas equivalentes são mostrados na Fig. 5.4. Pela dificuldade de se estimar com precisão o trem de cargas utilizado na análise experimental da viga, foram simulados dois limites de carregamento: um inferior e superior. Os limites inferior e superior dos trens de carga estão mostrados na Fig. 5.4.

O veículo se movimenta sobre a viga com uma velocidade constante de $10 \mathrm{~m} / \mathrm{s}(36 \mathrm{~km} / \mathrm{h})$.

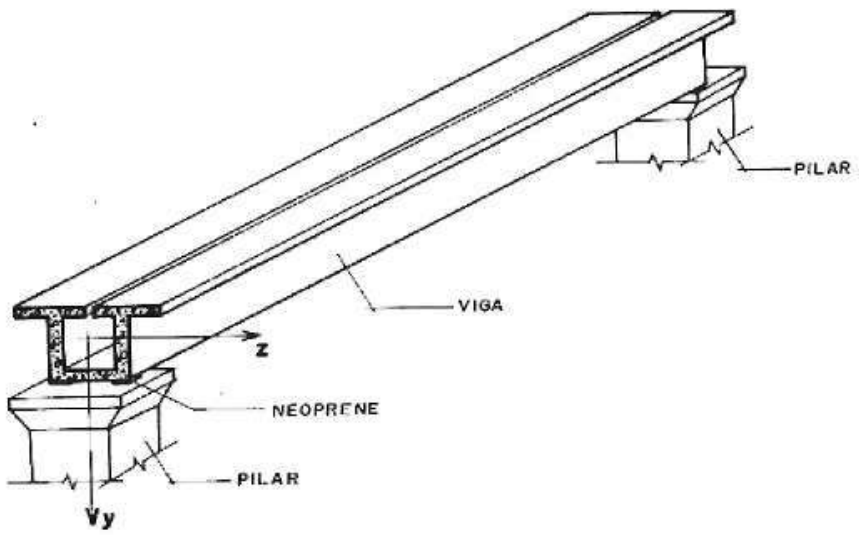

Fig. 5.3 - Viga reta elevada pertencente a um sistema de transporte urbano. (Exemplos B e F).
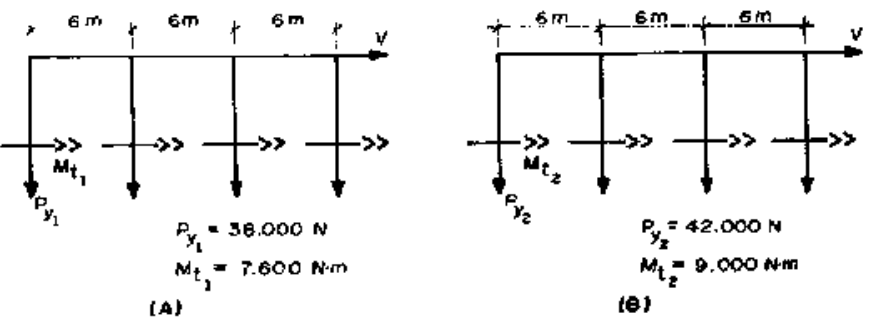

Fig. 54 - Trens de carga utilizados para representar o carregamento externo na viga reta

As curvas que representam os deslocamentos verticais teóricos e experimentais, no centro do vão da viga, referentes aos limites de carregamento teórico e ao carregamento real, respectivamente, estão mostradas na Fig. 5.5. Observa-se uma excelente correlação entre os resultados teóricos e o experimental, sendo que a diferença percentual entre a resposta teórica do deslocamento veritcal para o limite inferior do carregamento e a resposta experimental do deslocamento vertical é da ordem de 4\%; entre a resposta para o limite superior e a experimental, a diferença é da ordem de $2 \%$. 


\section{6 - SOBRE O COEFICIENTE DE IMPACTO EM PON- TES CURVAS DE CONCRETO}

Os efeitos dinâmicos induzidos pelas cargas móveis podem ser levados em conta em forma simples na análise estrutural, multíplicando-se a resposta estática da estrutura por um coeficiente de majoração (C.M.D.). Esse coeficiente é geralmente definido como a relação entre a resposta dinâmica máxima e a resposta estática correspondente a posição mais desfavorável das cargas.

Fig. 6.1 (a)

Fig. $6.1(\mathrm{~b})$
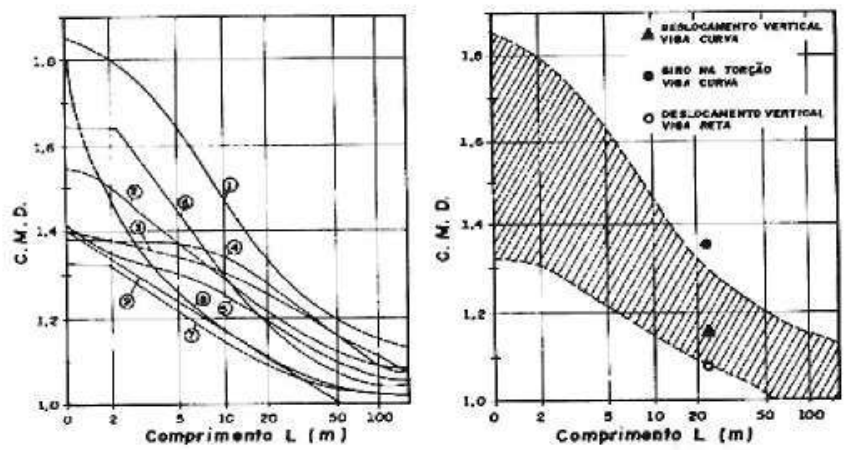

Fig. 6.1 (a) - Coeficientes de Majoraçāo Dinâmica dados pelas diferentes Normas para ponteg rodoviárias. 1,2 Suiģa 3 U.S.A.; 4 U.R.S.S.; 5 Suécia; 6,7 R.D.A.; 8 França; 9 Brasil (NB - 2)
Fig. 6.1 (b) - Coefficientes de Impacto (C.M.D.)

As recomendações de diversas normas sobre o coeficiente de majoração dinâmica, as vezes denominado fator de impacto, nem sempre são fundamentadas em sólidas considerações teóricas ou em ensaios sistemáticos. A Fig. 6.1 (a) mostra a grande dispersão dos C.M.D. sugeridos por diferentes normas. O quadro descrito está experimentando uma significativa evolução, devido à influência de estudos recentes sobre o tema, nos quais começam a ser incorporados os efeitos de interação veículo-via e das imperfeições inevitavelmente presentes na última. PALAMAS $^{6}$ (1984), por exemplo, apresenta um estudo detalhado sobre a influência das imperfeições na C.M.D.

É interessante sublinhar aqui que a existência de curvatura em planta, mesmo quando pequena, resulta usualmente num importante acréscimo dos coeficientes de impacto, como se observa na Fig. 6.1 (b), baseada nos resultados obtidos nos exemplos $\mathrm{F}$ e $\mathrm{G}$.

\section{7 - CONCLUSÕES}

Foram apresentadas soluções estáticas e dinâmicas de vigas curvas de seção aberta e parede fina sob cargas móveis, obtidas a partir das equações de Vlasov, mediante aplicações de diferenças finitas centrais. O método numérico implementado no programa VIGADIN visa o desenvolvimento de uma ferramente eficiente para aplicação em estudos de interação de sistemas veículo-via. A comparação das previsões do método com outras soluções e com observações experimentais permite conferir o grau de precisão e a confiabilidade do mesmo. Também baseados nos exemplos estudados, são apresentados conclusões relativas a influência da curvatura sobre o período fundamental e os coeficientes de impacto em pontes de concreto.

\begin{abstract}
This work deals with linear analysis of nonsymmetric curved thin-walled bridges or beams with open cross section, with physical properties, geometry and radius of curvature constant along the centroidal axes. VLASOV differential equations where used, considering the coupling between deformations on the vertical, transversal, axial, and torsional directions. Central finite difference method was used to solve the system of four partial differential equations. Bacisally two analysis were performed: DINAMIC and STATIC. Both were also used to obtain the impact coeficcient (C.M.D.). The first analysis refers to the determination of the basic dynamic characteristies (natural frequency and vibration modes), and the determination of the dynamic response of the beam, in stresses and deformations, for arbitrary mobile loads. Beams with any combination of the boundary conditions, including pinned or fixed ends on the three directions of torsion and bending were considered. A comparison between the results of the theoretical analysis, produced by computer programs impemented in a microcomputer (for the static analysis) and computer B-6700 (for the dynamic analysis), and the ones given in the references and also obtained experimentally. Showed a good correlation.
\end{abstract}

KEY WORDS: Curved Beams, Moving Loads, Natural Frequency.

\title{
REFERÊNCIAS BIBLIOGRÁFICAS
}

1 - CANTIENE, R. Dynamic loed testing of highway bridges. IABSE Proceedings, Zürich, (75).57-72, Aug. 1984.
2 - Carbonari, G. Andilise Dinämico de Vigas Assimétricas em Curva com Parede Fina e Aberta Submetida a Açāa de Cargas Móveis. Curso de Pós-Graduaçãa em Engenharia Civil, UFRGS, 1986. Tese (Mestrado). 
3 - CHRISTIANO, P.P. \& CULVER, C.G. Horizontelly eurved bridges subject to moving load. Journal of the Structural Division, Now York, ASCE, 95(8): 1616 43. Aug. 1969.

4 - CULVER, C.E. Natural frequencias of horizontally curved beams. Journal of the Structural Division, New York, ASCE, $93(2)$ : 189-203, Apr. 1967.

5 - GROEHS, A.G. \& DOS SANTOS, M.l.G. Análise dia vie do sistema Coester de trarisporte pneumático. Parte 1 Analisa numbrica. In: Colloquia 85. MEMÓAIAS "B". INGENIE RIA ESTRUCTURAL. Tomo III: 739-52. Buenos Aires (Argentina) Octubre 1985.

6 - PALAMAS, J. Comportament dynamique des ponts sours P'effet de charges roulantes. Construction Metallique, Puteaux, 21|3\}:35-43, Sept. 1984.
7 - PROJETO Aerombvel: ansilise da via. Porto Alegre, Eseola de Engenharia da UfRGS, 1984.

8 - PROJETO Aeromb́el: anfilise simulada da vía - estru ture fundacōes. Porto Alegre, Escola de Enganharia da UFRCS, 1985.

9 - SHORE, S. \& CHAUDHURI, S. Free vibration of horizontally curvad beams. Journal of the Structural Division, Naw York, ASCE, 98(3):793-6, Mar. 1972.

10 - VLASOV, V.Z. Thin-walled elartic beams. 2 ed. Seruselem, israed Progam for Seientific Translation, 1961.

11 - YOO. C.H. \& FEHRENBACH, J.P. Natural frequeneies of curved girders. Journal of the Engineering Mechantes Division, Now York, ASCE, 107(2):339-54, Apr. 1981.

Recebido para publicaçío em 28/3/89 


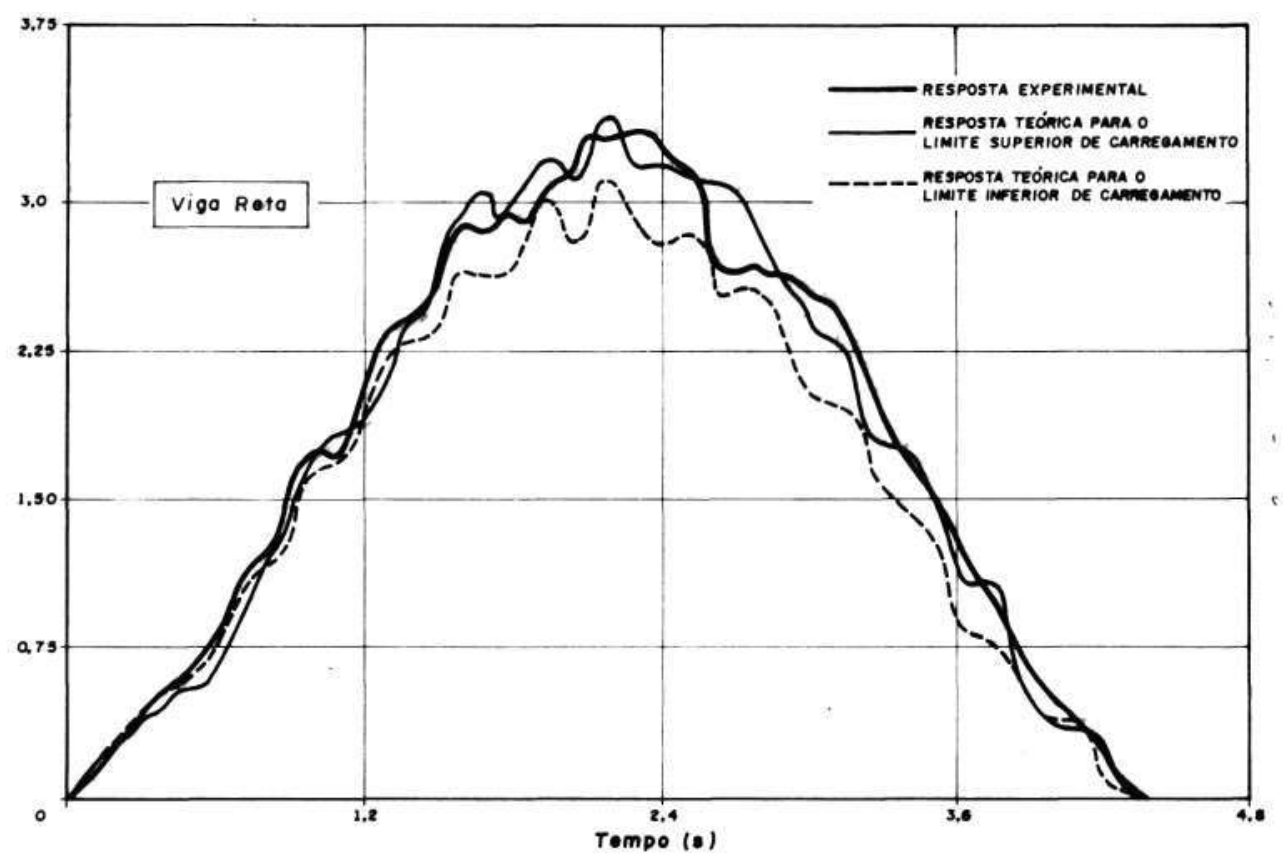

Fig. 5.5 - Resposta dinâmica da viga reta: Deslocamentos verticais no centro do vão.

Exemplo G: A viga analisada neste exemplo possui as mesmas características da viga estudada no Exemplo $\mathrm{F}$ desta seção, com a diferença de ser curva, com raio de curvatura constante e igual a $72 \mathrm{~m}$ (eqüivale a um ângulo interno de abertura da viga na ordem de 20 graus). $\mathrm{O}$ veículo se movimenta sobre a viga com veocidade de $20 \mathrm{~m} / \mathrm{s}(72 \mathrm{Km} / \mathrm{h})$, possuindo o trem de cargas mostrado na Fig. 5.6.

A resposta dinâmica da viga curva, em termos do deslocamento vertical e giro, está indicada na Fig. 5.7. Neste exemplo, fica saliente a influência do raio de curvatura no valor do C.M.D., ao se comparar com a resposta da viga reta. O C.M.D. máximo da viga curva, para o deslocamento vertical, é em torno de 1,14; no caso da torção, resulta igual a 1,34 .

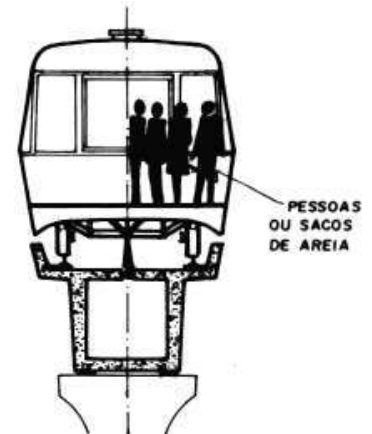

(A)

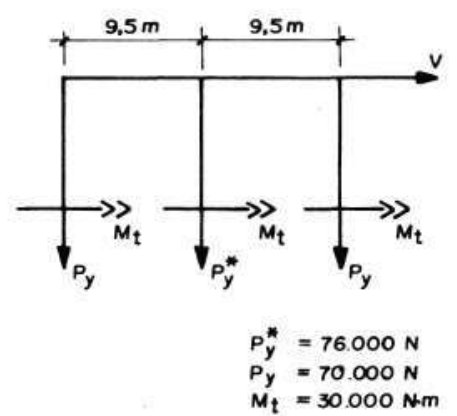

(B)
Fig. 5.6 - Carregamento externo do veículo em movimento sobre a viga reta. (Exemplo G).

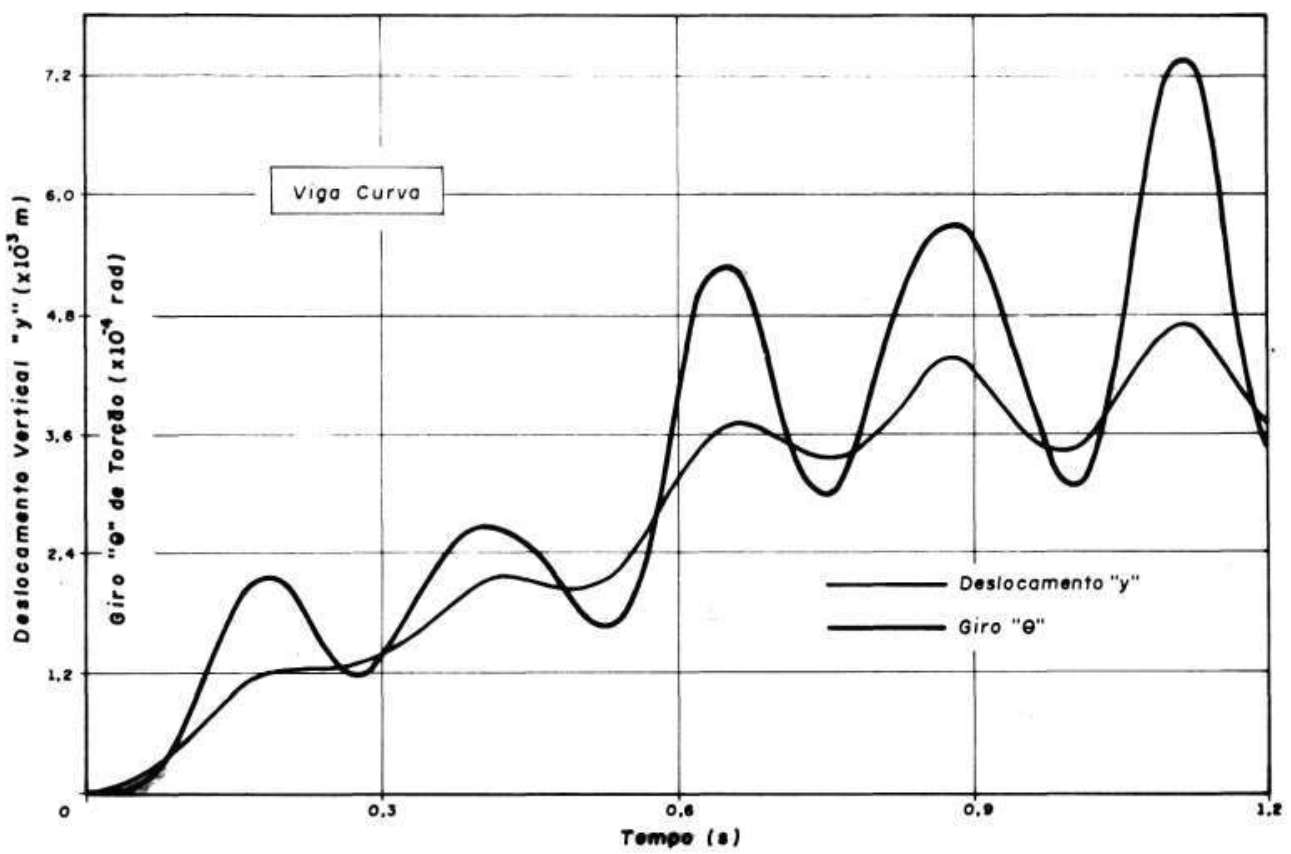

Fig. 5.7 - Resposta dinâmica da viga curva: Deslocamento Vertical e giro no centro do vão. (Exemplo G). 